\title{
Effect of friction and back pressure on the formability of superplastically formed aluminium alloy sheet
}

\author{
Paul Wood ${ }^{1,2, a}$, Muhammad Jawad Qarni ${ }^{1, b}$ and Andrzej Rosochowski ${ }^{2, c}$ \\ ${ }^{1}$ Advanced Forming Research Centre, University of Strathclyde, Glasgow, UK \\ ${ }^{2}$ Design, Manufacture and Engineering Management, University of Strathclyde, Glasgow, UK

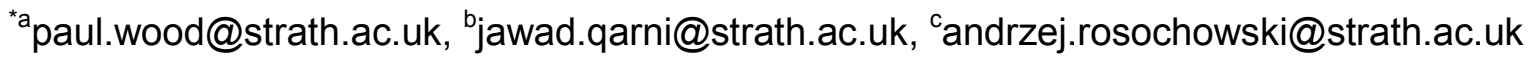 \\ * Corresponding author
}

Keywords: Aluminum alloy AA7475, FEA, Friction coefficient, Back pressure, Lubrication, Superplastic forming.

\begin{abstract}
This paper examines the effect of friction and back pressure on the formability of superplastically formed aluminium alloy AA7475 sheet at the temperature of $517{ }^{\circ} \mathrm{C}$. Several experiments with lubrication and back pressure are performed using a simple box shape tool cavity. The coefficient of Coulomb friction between the formed sheet and tool has been determined indirectly using a finite element model to simulate superplastic forming of the box shape. Typical values determined for all lubricant conditions tested are in the range $0.1<\mu<0.2$. The void growth with strain was determined directly from measurements as a function of back pressure. The results show the application of back pressure at $1 \mathrm{MPa}$ reduces the growth of voids from $7 \%$ to $0.3 \%$ void volume fraction at a logarithmic thickness strain of 0.65 . This paper reports back pressure has a significantly greater role than friction in enhancing the formability of the alloy.
\end{abstract}

\section{Introduction}

Superplasticity is the ability of a metal alloy to be permanently stretched to very high elongation for a certain combination of temperature and strain rate $[1,2]$. Superplastic forming (SPF) of sheet metal alloys uses the high ductility and relatively low forming pressures to manufacture components with intricate shapes in a single forming operation. Components of high structural integrity may be produced this way, enabling an assembly with fewer parts and reduced weight. SPF technology is used in high value products and small volumes. Although some aluminium alloys have found application in niche vehicles products in the automotive sector, the quantities are relatively low volume. The process and material cost remains a barrier to broader application of the technology across industry sectors.

The presence of contact friction between sheet and tool surface results in a thickness strain gradient in the formed component. The coefficient of Coulomb friction between SPF aluminium alloy sheet and tool material has been determined using test apparatus for direct measurement of friction [3]. However, details of the test apparatus used are not clearly described. Laboratory tests were performed both with and without lubrication (using graphite) for workpiece and tool material in contact at SPF temperatures, in both air and nitrogen atmospheres. The pressure applied between workpiece and tool was varied in the range 1 to $8 \mathrm{MPa}$. The coefficient of friction was measured for SPF aluminium grades AA7475 and AA5083, and steel and alumina based cement tool materials. It was found that the lubricant and atmosphere had little effect on the coefficient of friction. For the AA7475 alloy, the coefficient of friction decreases, typically from 0.2 to 0.1 , as normal pressure increases in the range tested. Another study [4] with AA8090 has reported Coulomb friction varies with position of sheet in the tool; the friction coefficient over the die entry radius and side walls is substantially higher than at the base of the tool.

Fine grain aluminium alloy sheet tends to form small cavities under superplastic deformation. The void volume fraction of the cavities increases exponentially with strain [2,5,6,7], and their growth rate is dependent on the stress state, temperature and strain rate. For AA7475, the higher forming temperature of $521{ }^{\circ} \mathrm{C}$ defers [5] the void growth to a higher strain $(\varepsilon>1)$; at the strain rate 
of $2 \times 10^{-4} \mathrm{~s}^{-1}$ this forming temperature gives the highest strain rate sensitivity exponent $m \cong 0.8$. The effect of strain rate on the rate of cavity growth in the SPF range is less significant than temperature. It has been reported that the rate of growth of cavities increases under an equi-biaxial tensile stress state over uniaxial tensile stress state for a range of aluminium alloys [7]. Biaxial tension increases the level of hydrostatic tensile stress. A compressive stress applied normal to the plane of the sheet using a hydrostatic pressure $(p)$ will decrease the rate of void growth with strain [6] in the material under a biaxial stress state if $p>0.75 \sigma_{e}$; where $\sigma_{e}$ is the equivalent uniaxial flow stress of the material at the optimum strain rate and forming temperature. Here $\sigma_{e} \cong 3 \mathrm{MPa}$ so that $p$ $>2 \mathrm{MPa}$.

In the present study, the formability of AA7475 (2 mm thick) at the SPF temperature of $517{ }^{\circ} \mathrm{C}$ is investigated. The experiments performed use a simple box shape tool cavity to study the effectiveness of a Boron Nitride lubricant to reduce friction between work piece and tool by varying the method of application of lubricant. In combination with the lubricant trials, back pressure is applied, which is varied at three levels. It is desirable to reduce both friction and back pressure to reduce process costs and improved utilisation of material in the end product.

\section{Experimental procedure}

The shape of the tool cavity to form the box is $300 \times 300 \mathrm{~mm}$ plan and $150 \mathrm{~mm}$ deep with a $2^{\circ}$ draft angle at the walls of the cavity. Further dimensions are given in Fig. 1. The overall tool plan size is $500 \times 500 \mathrm{~mm}$ and the tool material is H13 grade tool steel. The sheet thickness is measured at the specified locations (see Fig. 1) after forming.

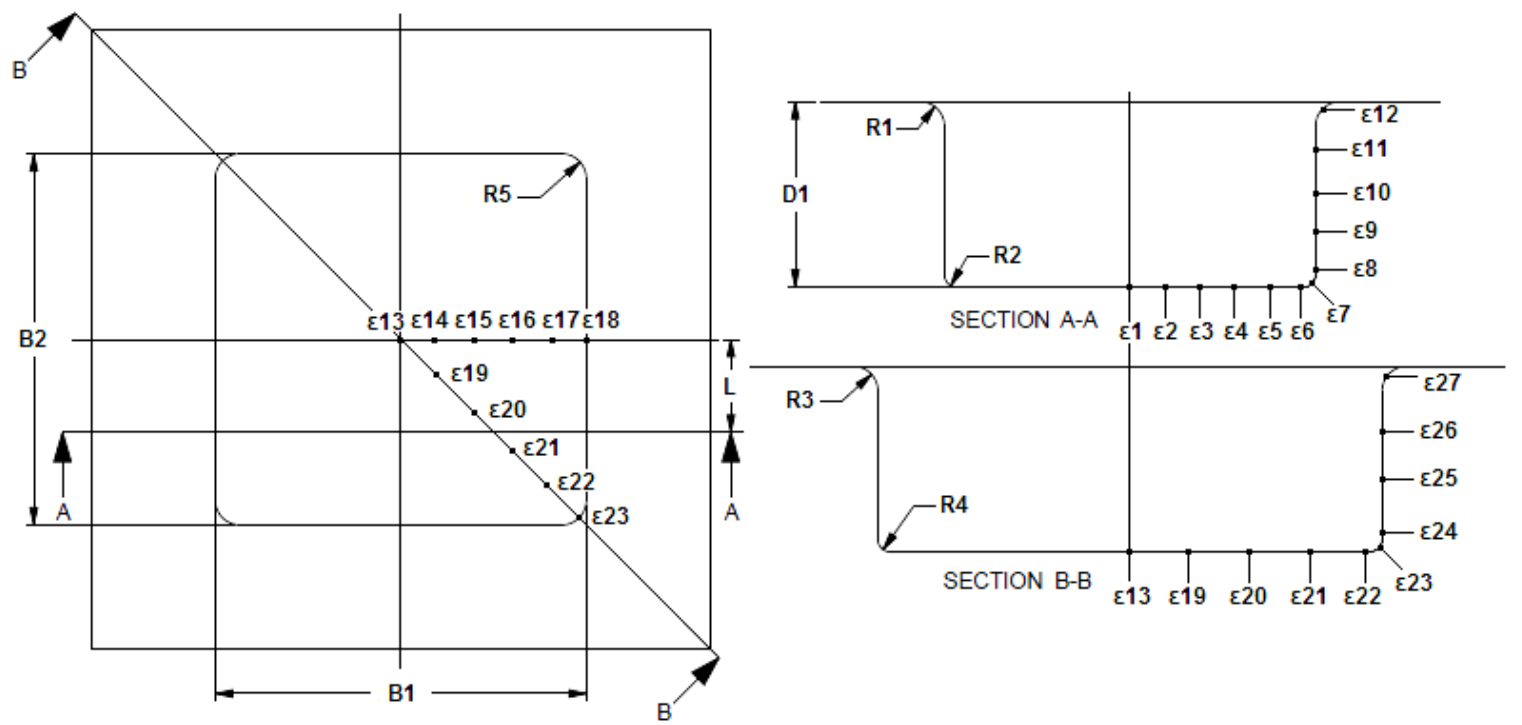

Dimension [in mm] of tool cavity

\begin{tabular}{rllllllll}
\hline B1 & B2 & D1 & L & R1 & R2 & R3 & R4 & R5 \\
\hline 300.0 & 300.0 & 150.0 & 85.0 & 15.0 & 4.0 & 15.0 & 4.0 & 25.0 \\
\hline
\end{tabular}

Figure 1: Geometry of the tool cavity showing locations for thickness measurements.

The experimental plan is summarised in table 1. In total three boxes are formed. The Boron Nitride is mixed with three parts of water. The method of application of lubricant in test no. 1 is a dust coat brushed onto the sheet before forming. In test no. 2 a dust coat is sprayed onto the sheet before forming. In test 3 a thick coat is sprayed onto the sheet and tool cavity surface before forming. 
Three levels of back pressure $\left(p_{b}\right)$ are studied; these are $p=0,1$ and $2 \mathrm{MPa}$. The $p_{b}$ is held constant in each experiment while forming is performed. A forming pressure $\left(p_{f}\right)$ is superimposed on the $p_{b}$ to create a pressure difference $\left(p_{d}\right)$ which is also common to each test undertaken so that $p_{f}$ $=p_{b}+p_{d}$. For example with $p_{b}=0$ then $p_{f}=p_{d}$; with $p_{b}=1 \mathrm{MPa}$ then $p_{f}(\mathrm{MPa})=1+p_{d}$. The typical $p_{d}$ required to form a box is $0.1 p_{b}$ at $1 \mathrm{MPa}$ and $0.05 p_{b}$ at $2 \mathrm{MPa}$. The $p_{f}$ and $p_{b}$ are applied using pressurised inert gas which is fed and controlled by two separate lines. The rate of application of $p_{f}$ will allow the strain rate to increase up to fivefold from an initial rate of $1 \times 10^{-4} \mathrm{~s}^{-1}$ as the sheet is formed into the tool cavity. To prevent gas leakage during forming, the tool has a small lip protruding from the top surface of the tool. The lip bites into the deformable sheet when sufficient clamp load is applied between seal plate and tool to minimise leakage at maximum pressure. The clamp load is sufficient to restrain the sheet at the lip to prevent additional material being pulled into the tool cavity. The temperature of the tool during experiments is held at $517 \pm 2{ }^{\circ} \mathrm{C}$.

Table 1: Experimental plan for the study.

\begin{tabular}{clcc}
\hline TEST No. & LUBRICANT \& METHOD & $\begin{array}{c}\text { BACK } \\
\text { PRESSURE }\end{array}$ & $\begin{array}{c}\text { FORMING } \\
\text { TEMPERATURE }\end{array}$ \\
\hline & BORON NITRIDE (DAG 5710) Mixed with 3 parts water & MPa & 'C \\
\hline 1 & Dust coat brushed onto sheet before forming (none on tool) & 0 & $517+/-2$ \\
\hline 2 & Dust coat spayed onto sheet before forming (none on tool) & 1 & $517+/-2$ \\
\hline 3 & $\begin{array}{l}\text { Thick coat spayed onto sheet before forming and tool cavity } \\
\text { surface during tool heat }\end{array}$ & 2 & $517+/-2$ \\
\hline
\end{tabular}

The test results obtained from the experiments are summarized in Table 2 . In the case of test 1 and 2 the shape of the formed box is different to the geometry of the tool cavity. This is because the sheet ruptured in the corner at R4 before the box had fully formed into the tool cavity.

Table 2: Measured dimension [in $\mathrm{mm}$ ] after SPF operation.

\begin{tabular}{lllllll}
\hline Test No. & R1 & R2 & R3 & R4 & R5 & Notes \\
\hline $\mathbf{1}$ & 15.0 & 12.0 & 15.0 & 30.0 & 32.0 & Sheet ruptured at R4 \\
\hline $\mathbf{2}$ & 15.0 & 8.0 & 15.0 & 15.0 & 30.0 & Sheet ruptured at R4 \\
\hline $\mathbf{3}$ & 15.0 & 4.0 & 15.0 & 4.0 & 25.0 & No rupture (fully formed) \\
\hline
\end{tabular}

The measured shape of the formed box in test no. 3 is consistent with the dimensions given in Fig. 1, because it had formed without rupture. The box in test no. 1 showed the greatest deviation between its final shape and the tool cavity. A combination of improved lubrication and higher back pressure is responsible for improving formability of the alloy under SPF conditions.

The strain distribution around the box is determined by measuring the thickness at each position identified in Fig. 1. The thickness strain is calculated using the equation [Eq. 1];

$$
\varepsilon=\ln \left(\frac{t_{i}}{t_{f}}\right)
$$

where $t_{i}=$ initial thickness and $t_{f}=$ final thickness of the sheet. The strain distribution in each formed box is given in Fig. 2. The graph shows that the strain gradient per unit length is steeper along the sides than along the base. 


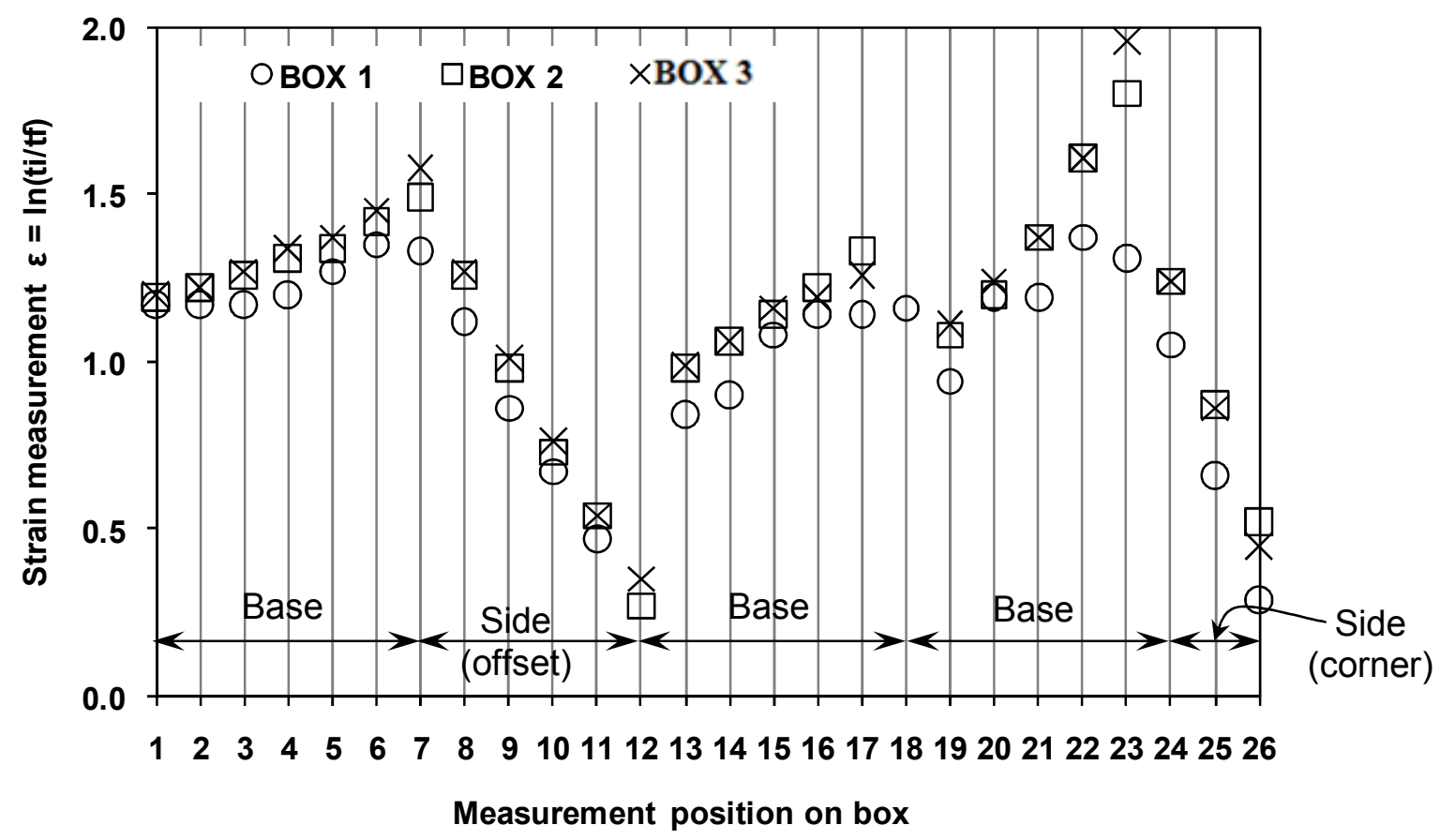

Figure 2: Thickness strain results for each formed box.

The void volume fraction in the material was measured in each formed box at various levels of thickness strain in the range $0.65<\varepsilon<1.3$. The Fig. 3 shows the effect of back pressure on the growth of voids with strain. The figure shows for box 1 with $p_{b}=0$ the void volume fraction attains $7 \%$ at $\varepsilon \sim 0.65$ and increases to a little over $10 \%$ at $\varepsilon \sim 1.3$. For the remaining test boxes 2 and 3 , the void volume fraction does not exceed $0.3 \%$ in the strain range measured.

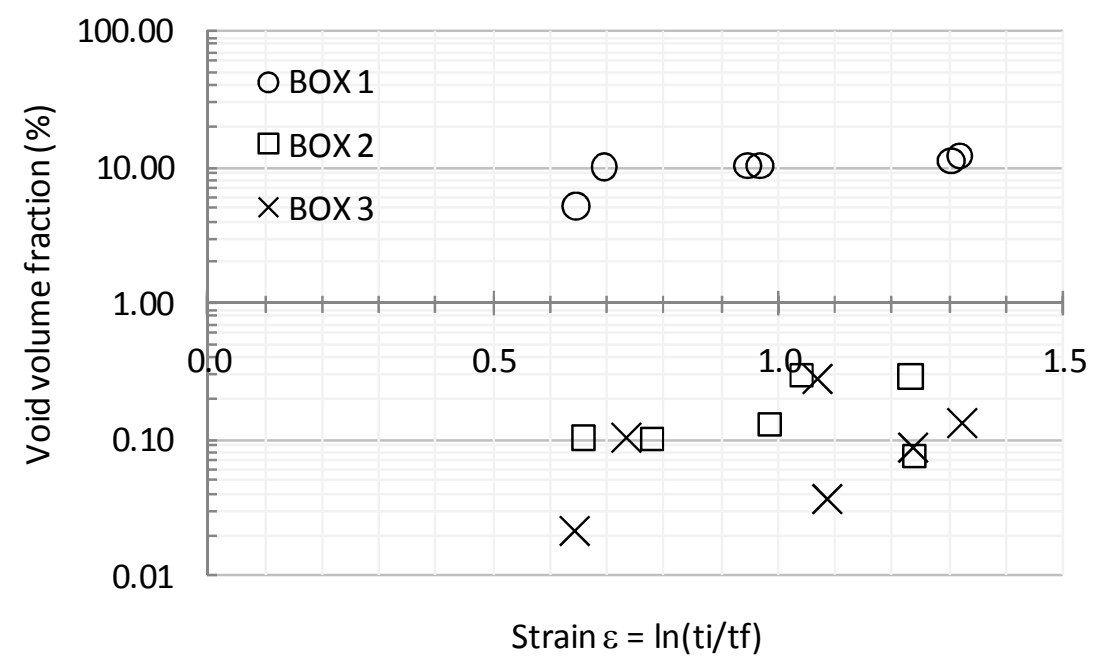

Figure 3: Effect of back pressure on the development of cavities with strain.

\section{Finite element modelling}

To investigate the effect of friction on the resulting strain gradient in each of the formed boxes a finite element (FE) model is developed using LS-DYNA[8] to simulate SPF of the box shape. The coefficient of Coulomb friction between the formed sheet and tool surface will be determined indirectly from the model, by reducing the error in the measured thickness strain distribution between model and test for each formed box.

The advantage of symmetry is utilized and only a quarter of the geometry is modelled in three dimensions to reduce computational time as shown in Fig. 4. The deformable sheet $(2 \mathrm{~mm}$ nominal thickness) is described using plane stress quadrilateral shell elements. The stress tensor normal to 
the plane of the shell element is zero for this element type. The shell element used is a reduced integration formulation with stiffness hourglass control to suppress the formation of hourglass modes. Five through thickness integration points describe the bending stiffness of the deformable shell element. The geometry of the tool surface is described in the FE model as shown in Fig. 1 and given a rigid material description.

A review of the material models under superplastic flow conditions is given in the paper [9]. The material in the finite element model in this paper is described by a viscoplastic constitutive hardening law of the form [Eq. 2];

$$
\sigma=k \varepsilon^{n} \dot{\varepsilon}^{m}
$$

The hardening constant $k$, strain hardening exponent $n$ and strain rate sensitivity exponent $m$, may each be a varying function of $\varepsilon, \dot{\varepsilon}$ and $\mathrm{T}$, where $\dot{\varepsilon}$ is strain rate and $\mathrm{T}$ is temperature. The parameters in the material model are fitted to test results derived under uniaxial tensile conditions in the strain rate and plastic strain range of interest [2].

The friction law used with the contact algorithm is Coulomb's formulation in which friction force is proportional to the normal force between the surfaces in contact [Eq. 3];

$$
F=\mu N
$$

where $F$ is the friction force, $\mu$ is the coefficient of friction and $N$ is the normal force between the surfaces in contact. Here $\mu$ is taken to mean sliding friction and not the resistance to overcome static friction for a body initially at rest which typically has a higher value. The Coulomb friction model may be applied until the shear stress $(\tau)$ of the work piece material is exceeded typically when $\tau>0.577 \sigma_{e}$. Above this threshold, the shear strength of the material offers lower resistance and contact friction is proportional to the flow strength of the material. Eight values of $\mu$ are investigated using the Eq. 3 in the FE model; these are 0.01, 0.02, 0.05, 0.1, 0.2, 0.3, 0.4 and 0.5.
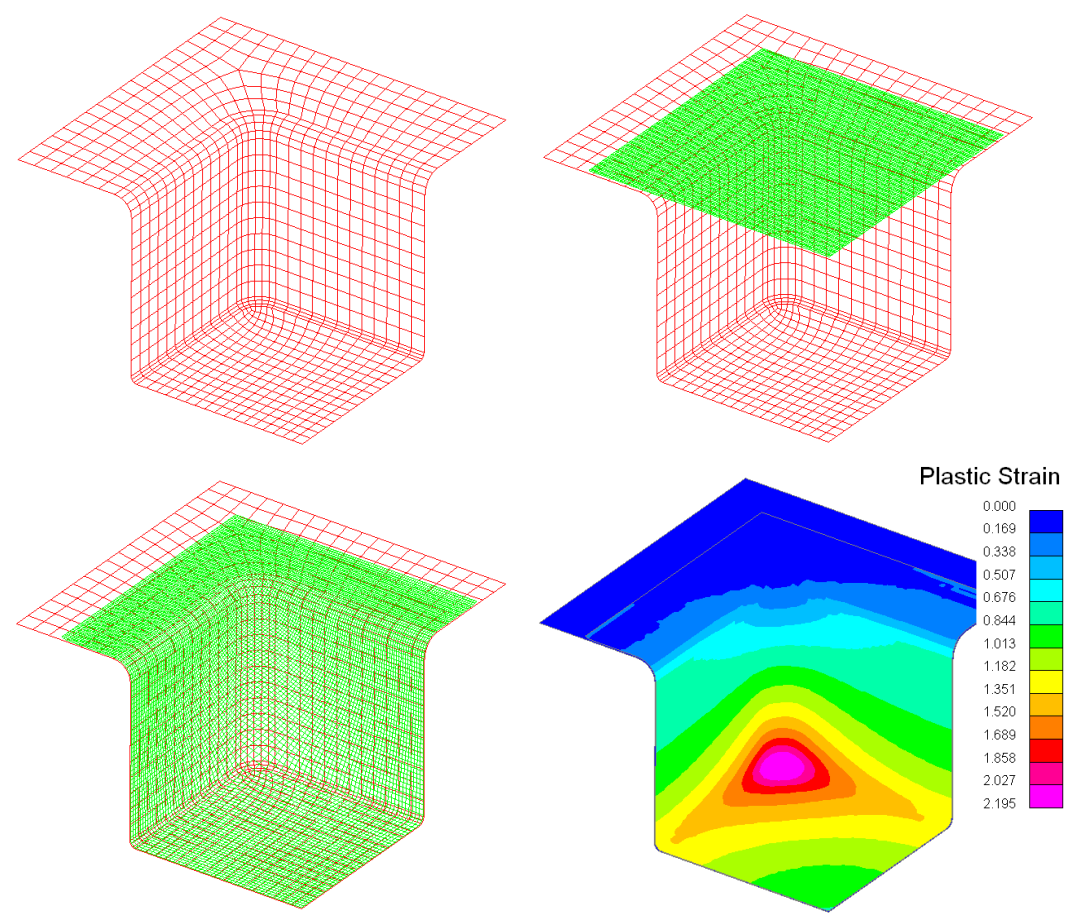

Figure 4: FE model of sheet and tool cavity (quarter symmetry used).

The forming simulation reproduced the differential pressure rate $\left(p_{d}\right)$ which is common to each back pressure $\left(p_{b}\right)$ used in the three experiments. Because the normal stress tensor of a plane stress shell element is zero there is no purpose in simulating $p_{f}$ and $p_{b}$ as separate pressure loads in the FE model. The total duration of the forming time was $12000 \mathrm{sec}\left(\sim 3 \frac{1 / 2}{\mathrm{hrs}}\right)$. The model was tested 
using explicit and implicit algorithms with $\mu=0.5$. Fig. 5 compares the equivalent plastic strain rate measured at a node in the corner of the deforming sheet. Although the difference in the result is not great, the explicit algorithm is used to support the wider numerical investigations.

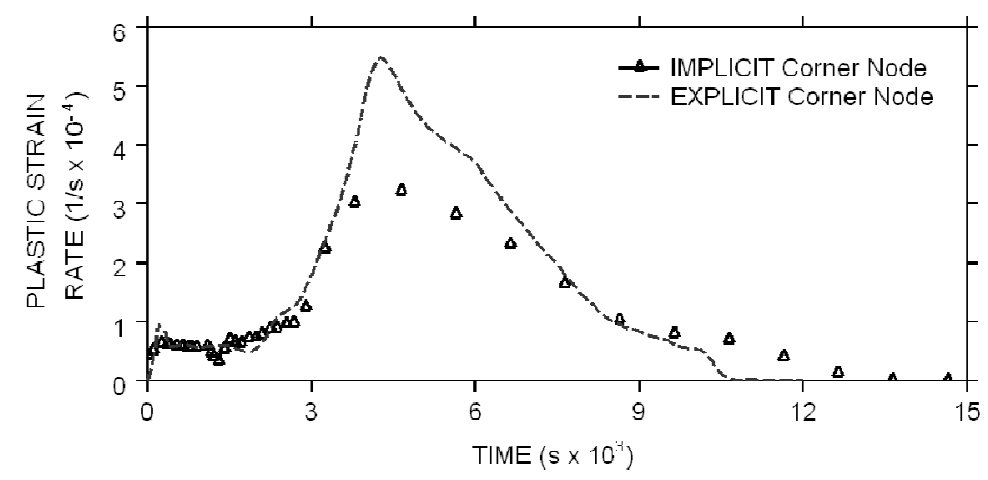

Figure 5: Comparing strain rate derived at a node in the forming sheet simulation for explicit and implicit methods using the differential pressure rate $\left(p_{d}\right)$ applied in experiments

\section{Results and discussion}

The distinguishing variable in the FE model is the value of $\mu$ used. The simulation of each test 1 , 2 and 3, was terminated so that the box shape formed corresponds to the measured geometry given in Table 2. For each of the three simulated box shapes, strain point measurements are determined at the same node position for each value of $\mu$.

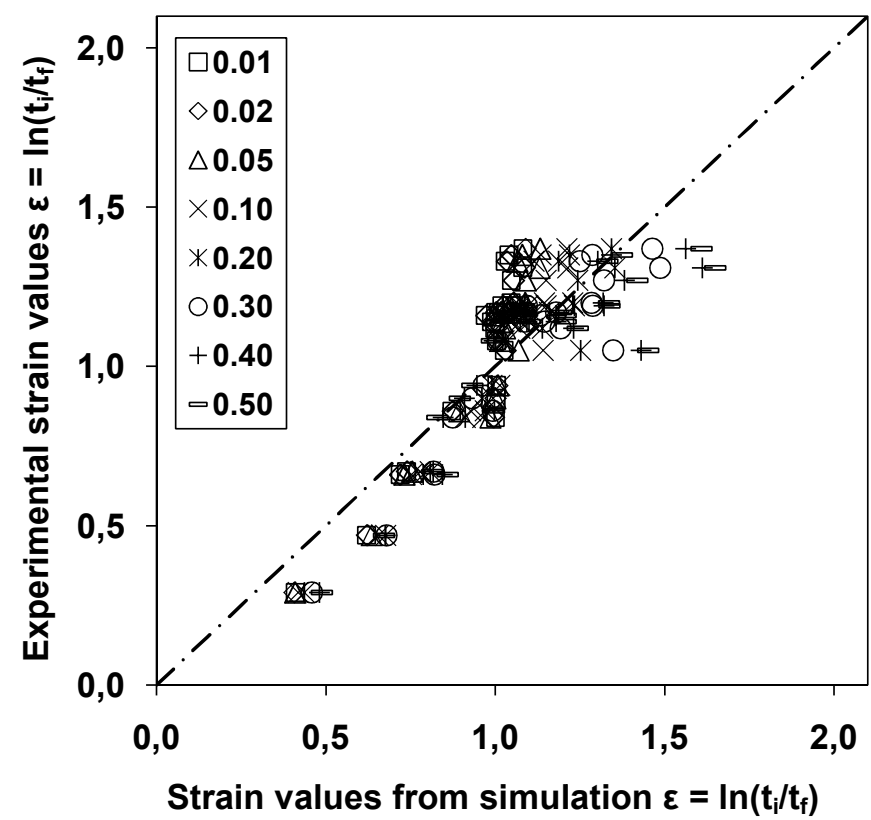

(a)

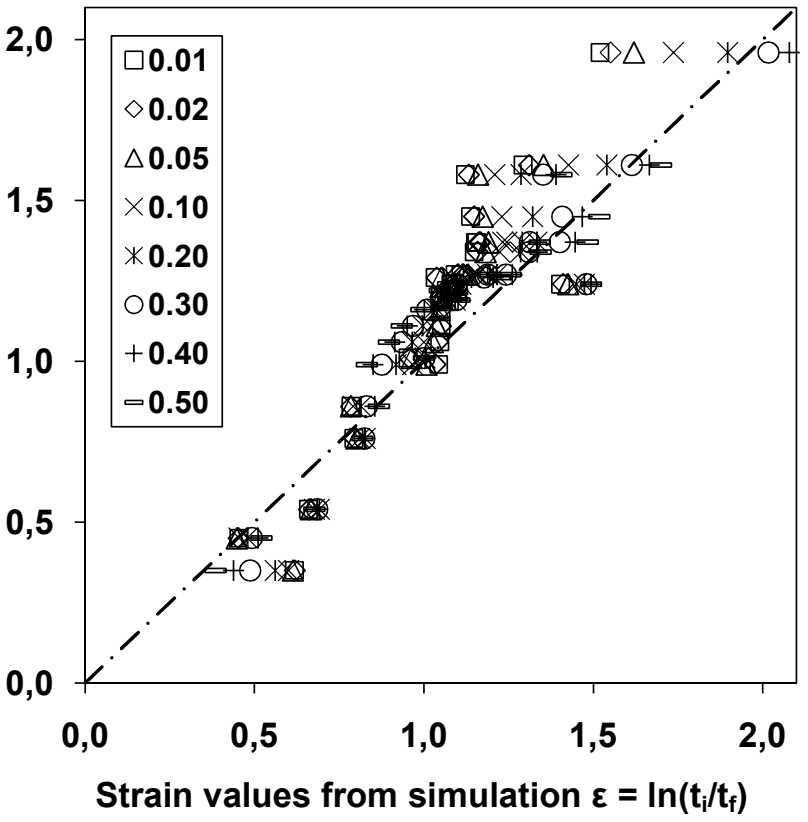

(b)

Figure 6: Comparing strain point measurements from experiments (a) Test 1 and (b) Test 3 with simulated results using different values of friction coefficient.

Fig. 6 compares strain point measurements obtained from the two of the three boxes formed in experiments with numerical results using different values of $\mu$. The abscissa is the numerical results and the ordinate is the experimental results. The legend in each graph identifies $\mu$ used in each model. 


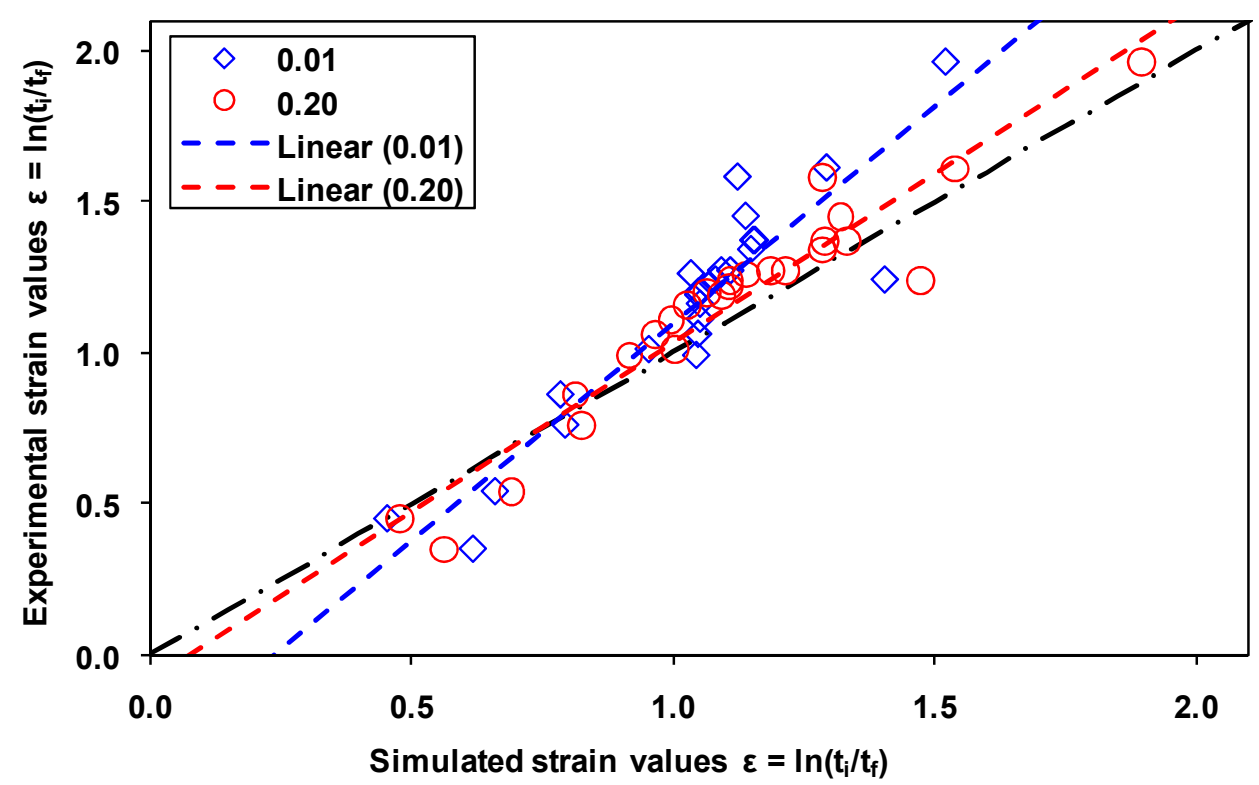

Figure 7: Correlation of strain point measurements between model and test box 3, and correspondence to line of ideal linear correlation.

The method to determine the friction coefficient uses linear correlation between strain point measurements obtained from the experiments and the simulation of each box. For box 3, Fig. 7 shows a line of best fit through the data for $\mu=0.01$ and 0.2 . It is observed that the line fitted to the data converges towards the line of ideal correlation as $\mu$ increases from 0.01 to 0.2 ; hence the error in strain point measurement decreases with increasing $\mu$. With $\mu>0.2$ it was found the error increases. For box 3, the minimum error in strain point measurement is given with $\mu=0.2$.

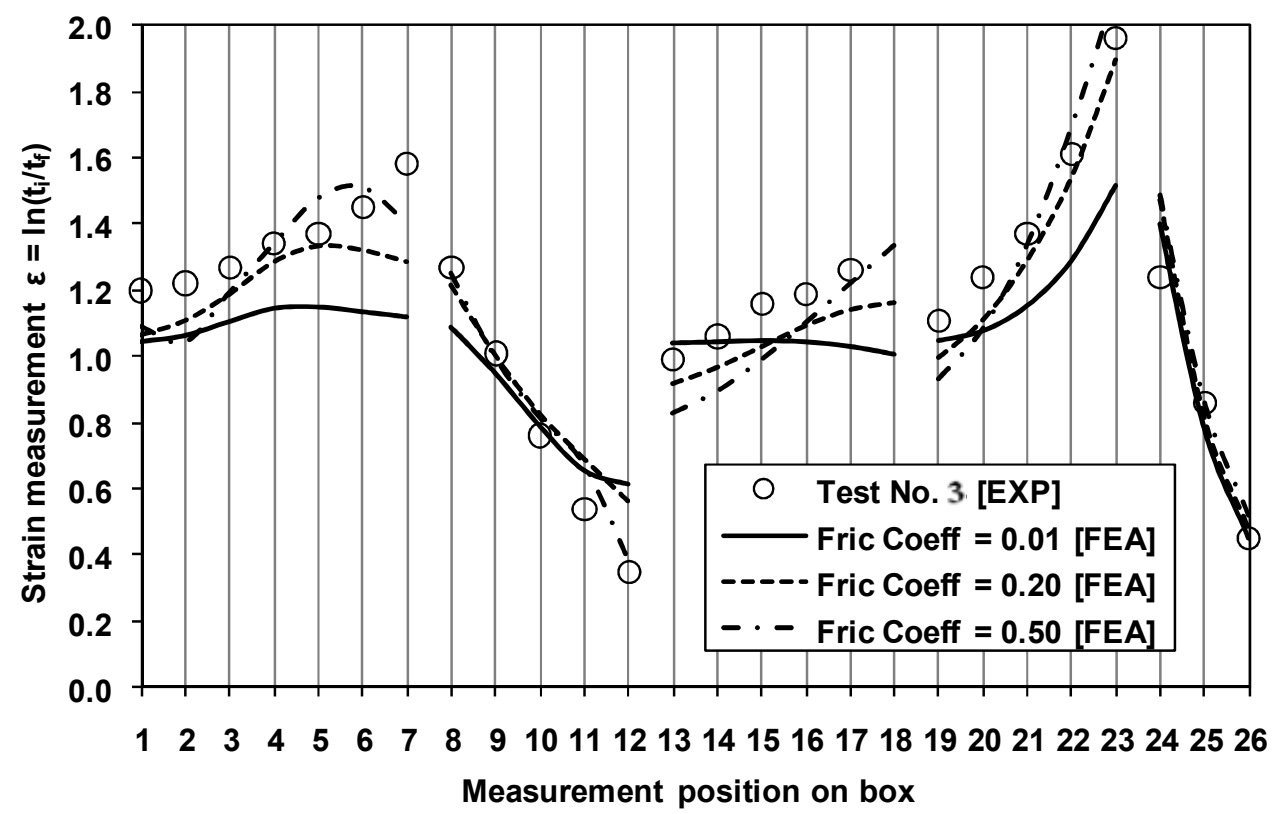

Figure 8: Comparing strain point measurements from test box 3 with simulated results for selected values of friction coefficient.

Fig. 8 compares strain point measurements from experiment no. 3 with simulated results for $\mu=$ $0.01,0.2,0.5$. The highest error is observed for $\mu=0.01$ and this generally occurs across the base sections of the formed box. The smallest error is with $\mu=0.2$ but increases only marginally for $\mu>$ 0.2 . 
The method of linear correlation is applied to the data of box 1 and 2 to determine the value of $\mu$ that gives the minimum error between model and test result for strain point measurements across each formed box. The table 3 summarises the results.

Table 3. Table of calculated friction coefficients

\begin{tabular}{lccc} 
& BOX 1 & BOX 2 & BOX 3 \\
\hline Coefficient of friction & 0.1 & 0.2 & 0.2
\end{tabular}

The results are surprising because it was expected the value of $\mu$ for box 3 would give the lowest value resulting from the lubrication method used to reduce friction between workpiece and tool, and box 1 would give the highest value. Although $0.1<\mu<0.2$ is in agreement with the findings reported in [3] in which $\mu$ was determined by direct measurement and the lubricant studied (graphite) had little effect on $\mu$.

\section{Conclusions}

The results confirm that back pressure has a significantly greater effect than friction in enhancing the formability of the sheet alloy material. A higher level of back pressure reduces the rate of growth of voids with strain in the material, thereby allowing greater thickness strain in the formed box.

A more elaborate FE method in which back pressure is included in the model may yield different values for $\mu$. The FE model should include a void growth rate function to describe the material behaviour as a function of stress state and strain. Such a material model would require the use of continuum (or brick) elements with eight corner nodes to model the sheet material and this demands high computational cost. Nevertheless the simplified approach to modelling adopted in this paper using plane stress shell elements with 4 corner nodes to describe the sheet material is typical of industrial practice. Hence to simulate the superplastic forming of AA7475 using a Boron Nitride lubricant a suggested value for $\mu>=0.1$ to a first approximation. With $\mu<0.1$ the error increases significantly.

\section{References}

[1] T.G. Langdon: Metall. Trans. 13A (1982).

[2] C.H. Hamilton and A. K. Ghosh: Metals Handbook, 1988.

[3] Z. Chen and P.F. Thompson: Wear 201, 1996.

[4] T.R. Chen, J.C. Huang, Y.M. Hwang and T.D. Wang: Materials Science Forum, Vols 304306, 1999.

[5] S.P. Agrawal and J.M. Tuss: Northrop Corporation, Aircraft Division, Hawthorne, California 90250.

[6] N. Ridley and J. Pilling: University of Manchester/UMIST, Manchester, UK.

[7] D.H. Bae, A.K. Ghosh and J.R. Bradley: Metallurgical and Material Transactions A, Vol. 34A, 2003.

[8] J.O. Hallquist: Version 971, 2007, Livermore Software Technology Corporation, 7374 Las Positas Road, Livermore, California 94551, ISBN 0-9778540-2-7.

[9] H.L. Xing, C.W. Wang, K.F. Zhang and Z.R. Wang: Journal of Materials Processing Technology Vol 151 (2004), p. 196-202. 
Sheet Metal 2011

doi:10.4028/www.scientific.net/KEM.473

Effect of Friction and Back Pressure on the Formability of Superplastically Formed Aluminium Alloy Sheet

doi:10.4028/www.scientific.net/KEM.473.532 